\begin{tabular}{l} 
Bentham OPEN \\
CrossMark \\
\hline
\end{tabular}

\title{
Managing Primary Angle Closure Glaucoma - The Role of Lens Extraction in this Era
}

\author{
Nafees Baig ${ }^{1,2}$, Ka-Wai Kam ${ }^{1,3}$ and Clement C.Y. Tham ${ }^{1,3, *}$ \\ ${ }^{I}$ Department of Ophthalmology \& Visual Sciences, The Chinese University of Hong Kong, Hong Kong SAR, People's \\ Republic of China \\ ${ }^{2}$ Hong Kong Eye Hospital, Kowloon, Hong Kong SAR, People's Republic of China \\ ${ }^{3}$ Prince of Wales Hospital, Shatin, N.T., Hong Kong SAR, People's Republic of China
}

\begin{abstract}
Trabeculectomy has been the gold standard in reducing intraocular pressure (IOP) in glaucoma patients, no matter it is angle closure or open angle glaucoma. However in primary angle closure glaucoma, no matter the lens is cataractous or not, it is likely to be pathological, this thicker than usual lens, with or without a more anterior position, is often regarded as a strong contributing factor to angle closure. Lens extraction, no matter it is cataractous or clear, can theoretically eliminate this anatomical predisposing factor of angle closure, and thus IOP can be reduced. Based on recent results of a number of clinical trials, lens extraction alone or in combination with other IOP-lowering surgeries, may therefore play a more important role in the treating primary angle closure glaucoma. In cases when greater IOP-lowering effect is needed or if drug dependency has to be minimized, combined procedures, such as phacotrabeculectomy, can be considered, but the surgical risk can be higher than lens extraction alone.
\end{abstract}

Keywords: Acute primary angle closure, lens extraction, primary angle closure glaucoma, trabeculectomy.

Primary angle closure glaucoma (PACG) occurs when there is appositional or synechial closure of the iridocorneal angle in the absence of a specific ocular disease, leading to a blockage of aqueous outflow, raised intraocular pressure (IOP) and eventually glaucomatous optic neuropathy and visual field defect.

The initial treatments for PACG aim at eliminating pupil block and re-opening of appositional closure of the drainage angle; options include laser peripheral iridotomy (LPI) [1], or occasionally surgical peripheral iridectomy, and argon laser peripheral iridoplasty (ALPI) [2,3]. If re-opening appositional closure fails to control IOP, surgical options such as trabeculectomy with or without antimetabolite, goniosynechialysis (GSL), trabeculotomy, glaucoma drainage device implantation, and cyclodestructive procedures by laser or cryotherapy can be considered.

\section{TRABECULECTOMY IN PRIMARY ANGLE CLOSURE GLAUCOMA}

Trabeculectomy has been the gold standard in controlling IOP in glaucoma patients, irrespective of the angle status or the baseline IOP. Trabeculectomy is, however, associated with various potentially serious intraoperative and postoperative complications, such as hypotony, choroidal detachment, maculopathy, cataract development or progression, and bleb related complications.

Salmon reported one of the earliest results of trabeculectomy in primary angle closure glaucoma [4]. He retrospectively examined the outcomes of trabeculectomy in advanced medically-uncontrolled chronic angle closure glaucoma in 46 eyes of 39 patients during a 5-year period. The surgery was not associated with complications such as visual loss or malignant glaucoma. The IOP was successfully reduced to less than $21 \mathrm{mmHg}$ in 30 eyes (66.7\%) without medication, and in 11 eyes (24.4\%) with topical glaucoma medication, after a mean follow-up period of 19 months.

\footnotetext{
* Address correspondence to this author at the Department of Ophthalmology \& Visual Sciences, The Chinese University of Hong Kong, Hong Kong Eye Hospital, 147K Argyle Street, Kowloon, Hong Kong, Tel: (852) 3943 5823; Fax: (852) 2816 7093; Email: clemtham@cuhk.edu.hk
} 
Four patients(8.9\%) received a second trabeculectomy at a mean period of 35.8 months, and nine patients received a cataract extraction with intraocular lens implantation at a mean period of 23.9 months after the initial surgery. He concluded that despite trabeculectomy was effective and relatively safe in managing advanced chronic angle closure glaucoma, one third of the patients required a second operation (either a second trabeculectomy or a cataract extraction) within 3 years. With the advancement in surgical techniques and the use of antimetabolite adjuvants in trabeculectomy, we expect better outcomes in more recent studies.

Sihota et al. also retrospectively evaluated and compared the long-term results of trabeculectomy without antimetabolite in primary open angle glaucoma (POAG) and primary angle closure glaucoma (PACG) in an Asian population with at least 5-year follow up [5]. Among the sixty-four eyes of 64 patients, the qualified and absolute success rates for IOP control showed no significant difference between POAG and PACG eyes. However, twelve of PACG eyes (32\%) had a two-line decrease in visual acuity in contrast to four POAG eyes (15\%). This was explained by the progression or development of cataract. They concluded that trabeculectomy without antimetabolite appeared to be effective in lowering IOP and in preserving visual field over a period of 10 years in both POAG and PACG. However, the development or progression of cataract especially in eyes with PACG was also considered as an important postoperative issue.

Tsai et al. had retrospectively compared the long-term results of combined trabeculectomy and cataract extraction versus trabeculectomy in 99 Chinese PACG patients [6]. Seventy-five of them received combined surgery and twentyfour had trabeculectomy alone. The mean follow-up periods in the combined group and trabeculectomy group were $25.8+/-10.8$ months and 31.4+/- 8.9 months, respectively. Survival analysis showed that 3-year complete success rate was $56 \%$ in the combined group and $54 \%$ in the trabeculectomy group ( $p=0.903)$; while complete success was defined as either a $>20 \%$ reduction in IOP, or an IOP maintained at less than $15 \mathrm{mmg}$, without any medication. The IOP or number of medications between the 2 groups of patients showed no significant differences throughout the 3-year follow-up. The postoperative complications had similar incidences between groups $(p=0.232)$. Patients in the combined group did not require additional IOP-lowering surgical procedures, while $13(54 \%)$ eyes in the trabeculectomy group required either cataract extraction or further IOP-lowering surgical procedures $(p<0.001)$.

They concluded that the long-term IOP-lowering effect and surgical complications of both groups were comparable. However, combined surgery would require fewer subsequent surgical interventions.

Chen et al. retrospectively analyzed the results of trabeculectomy in 52 patients with either acute or chronic form of PACG [7]. They reviewed 15 patients with acute angle closure and 37 patients with chronic angle closure glaucoma. The mean follow-up period was 32 months (range, 26-42 months). When they compared the visual acuity loss after surgery, final IOP control and complication of bleb encapsulation between these 2 groups, the acute angle closure group appeared to perform significantly worse $(P=0.02, P<0.01, P=0.02$ respectively, Fisher exact test $)$ than the chronic PACG group. They concluded that trabeculectomy might not have as good results in patients with acute angle closure when compared with chronic PACG, because it might result in worsened visual acuity, higher failure rate and complications rate.

Based on the evidence, although trabeculectomy is effective in lowering IOP and hence preventing or slowing down disease progression in eyes with chronic PACG, it is associated with a considerable risk of post-operative cataract development or progression, which leads to a decrease in visual acuity that may influence patient's daily living or even resulting in additional surgery.

\section{LENS EXTRACTION IN PRIMARY ANGLE CLOSURE GLAUCOMA}

A characteristically shallower anterior chamber is often seen in PACG eyes, leading to a closed drainage angle [8 - 14]. PACG eyes have significantly thicker lens, with a steeper anterior surface, which is more often anteriorly positioned when compared with controls $[9,11]$. Lens extraction deepens the anterior chamber, widens the drainage angle, and reverses the anatomical predisposing factors of angle closure. A recent study with ultrasound biomicroscopy (UBM) measurements, has shown that the mean anterior chamber depth was increased significantly from 1799 to 3528 microns by cataract extraction alone, similarly the mean angle opening distance at 500 microns from scleral spur (AOD 500) was increased significantly from 208 to 468 microns after cataract extraction in PACG eyes [15].

In another study, the changes in the anterior chamber angle configuration, measured by UBM, were compared after combined trabeculectomy and cataract extraction with those after trabeculectomy alone in PACG eyes [16]. It concluded that in the eyes that underwent combined procedure, the ciliary body processes were more posteriorly 
positioned and the anterior chamber angle was significantly widened after the surgery, as compared with those underwent trabeculectomy alone. The posterior shift of the ciliary processes after combined procedure was indirect evidence for the important role of the lens in anterior chamber angle configuration in eyes with PACG.

Based on such evidence, lens extraction may be an important surgical option in managing PACG, either as an initial treatment, or can be performed subsequently when IOP is not controlled or appositional angle closure cannot be eliminated by initial options $[17,18]$.

Wishart and Atkinson were among the earliest groups who recommended cataract extraction as the first procedure in eyes with cataract and PACG, rather than combined cataract and filtering surgery [19]. They prospectively followed up twenty-three eyes of 22 patients with PACG who underwent extracapsular cataract extraction (ECCE) with intraocular lens (IOL) implantation to assess the effect of cataract extraction on IOP control, and they also compared them with a 21 eyes with open angle glaucoma (OAG) which also underwent ECCE. Among the PACG eyes, there was a low incidence of acute post-operative IOP elevations (9\%), and most of them $(65 \%)$ achieved an IOP less than 21 $\mathrm{mmHg}$. In the OAG group, however, the IOP control was mainly unaffected.

Lai et al. prospectively followed up 21 eyes with PACG and co-existing cataract of 21 patients who had phacoemulsification with primary intraocular lens implantation for a mean period of $20.7+/-3.6$ months [20]. Intraocular pressure was significantly decreased from a preoperative level of $19.7+/-6.1 \mathrm{mmHg}$ to $15.5+/-3.9 \mathrm{mmHg}$ at final follow-up $(P=0.022)$. The number of glaucoma medication was decreased significantly from a preoperative value of $1.91+/-0.77$ to $0.52+/-0.87$ at final follow-up $(P<0.001)$. Visual acuity improved significantly after surgery in 10 eyes (47.6\%), remained the same in 9 eyes $(42.9 \%)$ and deteriorated significantly in 2 eyes $(9.5 \%)$. The authors concluded that in primary angle closure glaucoma patients with co-existing cataract, cataract extraction alone (by phacoemulsification) could reduce both IOP and the number of glaucoma medication required significantly.

\section{LENS EXTRACTION AS INITIAL TREATMENT TO RE-OPEN APPOSITIONAL ANGLE CLOSURE}

Theoretically speaking, lens extraction could effectively eliminate pupil block and appositional angle closure in susceptible eyes, however there is, meanwhile, no published clinical data proving that lens extraction is a superior treatment option than LPI for PACG eyes at initial presentation. This draws attention to an on-going study, the EAGLE study, which is a multi-centered randomized controlled trial, designed to compare clear lens extraction with LPI as the initial treatment in newly diagnosed PACG eyes [21]. If cataract is present at initial presentation of PACG, early cataract extraction may be more readily justified as an initial option to widen up the drainage angle. If the lens is not cataractous, the future results of the EAGLE study will be essential to justify or not to justify a clear lens extraction.

\section{LENS EXTRACTION AS SUBSEQUENT TREATMENT AFTER PERIPHERAL IRIDOTOMY IN PACG EYES WITH CATARACT}

As discussed above, trabeculectomy alone has a limited role in PACG eyes with coexisting cataract, it is because trabeculectomy may accelerate cataract progression, this causes further shallowing of the anterior chamber, and therefore aggravating progressive angle closure. Man et al. [22] had published their results of UBM measurements before and after clear lens extraction and trabeculectomy for PACG eyes. The mean anterior chamber depth (ACD) measured by UBM was significantly increased from $1,983.8 \pm 176.8$ microns to $3335.0 \pm 174.2$ microns $(p<0.001)$ by clear lens extraction $(\mathrm{n}=26)$, but decreased from 2,000.2 \pm 214.5 microns to $1975.8 \pm 218.2$ microns ( $p=0.001)$ by trabeculectomy $(n=24)$.

Tham et al. has conducted two randomized controlled trials to compare phacoemulsification with combined phacotrabeculectomy in PACG eyes with co-existing cataract after LPI, where their glaucoma is either medically controlled, [23] or uncontrolled [24].

In the trial involving medically controlled eyes, phacoemulsification alone caused a $10.4 \%$ reduction in mean IOP (the mean pre-operative IOP was $16.3 \mathrm{mmHg}$ and the mean post-operative IOP was $14.5 \mathrm{mmHg}$ ) and a reduction in the mean number of glaucoma drugs by $59.2 \%$ (the mean number of drugs reduced from 2.2 pre-operatively to 1.1 postoperatively), over 24 months [23]. On the other hand, combined phacotrabeculectomy resulted in a $20.9 \%$ reduction in mean IOP (the mean pre-operative IOP reduced from $16.3 \mathrm{mmHg}$ to $13.3 \mathrm{mmHg}$ post-operatively) and a reduction in the mean number of glaucoma drugs by $95.2 \%$ (the mean number of drugs reduced from 2.3 pre-operatively to 0.1 postoperatively), over 24 months [23]. Combined procedure when compared with phacoemulsification, resulted in an additional $1.2 \mathrm{mmHg}$ reduction in mean IOP $(p=0.12)$ and a further reduction in the mean number of drugs $(1.0)$ 
$(p<0.001)$, over 24 months [23].

In the trial involving medically uncontrolled eyes, phacoemulsification alone resulted in a $34.4 \%$ reduction in mean IOP (the mean pre-operative IOP was reduced from $24.4 \mathrm{mmHg}$ to $16.1 \mathrm{mmHg}$ post-operatively) and a decrease in mean number of drugs by $52.4 \%$ (the mean number of drugs was 3.3 pre-operatively and 1.7 post-operatively), over 24 months [24]. Whereas phacotrabeculectomy resulted in a IOP reduction by $41.2 \%$, (the mean pre-operative IOP was reduced from $23.8 \mathrm{mmHg}$ to $14.1 \mathrm{mmHg}$ post-operatively) and a decrease in mean number of drugs by $89.6 \%$ (the mean number of drugs was 3.1 pre-operatively and 0.5 drugs post-operatively), over 24 months [24]. Combined procedure when compared with phacoemulsification, resulted in an additional $2.0 \mathrm{mmHg}$ lowering in mean IOP $(P=0.08)$ and a further reduction of 1.25 in the mean number of drugs $(P<0.001)$, over 24 months [24].

Combined phacotrabeculectomy was, however, associated with significantly more complications than phacoemulsification (complication rate in combined was $26.2 \% v s 8.1 \%$ in phacoemulsification) $(p=0.007)$ [25]. There were more surgical interventions performed to handle complications after combined phacotrabeculectomy than phacoemulsification alone (13.1\% vs $1.6 \%$ respectively, $p=0.01)$ [25]. At mean follow up period of 32.6 months, the changes in visual acuity, the rates of progression of glaucomatous optic neuropathy and visual field loss were not significantly different between the 2 treatment groups.

Based on the above evidence, it should be logical to consider performing cataract extraction alone in medically controlled PACG eyes with cataract, as combined surgery may result in additional risk of complications which may not be justified for the small additional clinical benefit. In contrary, in medically uncontrolled eyes with co-existing cataract, the risks of additional complications and interventions after phacotrabeculectomy may or may not be justified despite of its greater clinical benefits. Therefore, it is suggested that either approach could be considered depending on patient and ocular factors. Phacoemulsification alone may be preferred if, for example, the patient could not accept the risk of filtration complications, or if the eye is more vulnerable to the stress from combined surgery (e.g. very advanced glaucoma or poor corneal endothelium). On the other hand, combined surgery may be more favourable if, for example, the patient is known to have poor compliance or tolerance to his/ her medications, or difficult access to long-term medication or follow up.

Combined phacoemulsification with goniosynechialysis (GSL) can be an alternative to phacotrabeculectomy in PACG eyes. Teekhasaenee and Ritch had conducted a prospective non-controlled clinical trial which showed that combined phacoemulsification with GSL (phaco-GSL) and implantation of posterior chamber intraocular lens was effective in opening up peripheral anterior synechiae (PAS) and reducing IOP as well as improving visual acuity in chronic PACG eyes when the surgery was performed within 6 months after the treatment for acute angle closure (AAC) [26]. Phaco-GSL was performed in 52 eyes of 48 patients with uncontrolled IOP and more than 180 degrees of PAS, within 6 months after acute AAC. Among the 52 eyes, 47 of them (90.4\%) achieved an IOP less than 20 mmHg without medication, 4 of them had controlled IOP with medications, and only 1 eye needed subsequent filtration surgery. The mean extent of PAS reduced from 310 to 60 degrees, which was compatible with another case report [27] where ultrasound biomicroscopy (UBM) demonstrated a restoration of an open drainage angle after a successful GSL.

Tang et al. retrospectively compared the effectiveness of combined phacoemulsification and viscogoniosynechialysis (phaco-GSL) with trabeculectomy alone in PACG with coexisting cataract [28]. They analyzed 19 patients who underwent phaco-GSL and 20 patients who underwent trabeculectomy. The median follow-up periods were similar for both groups (10 months). At the last follow-up, the mean IOP was $14.09 \pm 6.89 \mathrm{mmHg}$ and the mean number of antiglaucoma medications was $0.36 \pm 0.59$ in phaco-GSL group; in trabeculectomy group, the mean IOP was $16.48 \pm$ $4.74 \mathrm{mmHg}(p=0.066)$ and the mean number of anti-glaucoma medications was $1.05 \pm 0.99(p=0.025)$. In terms of the mean central anterior chamber depth and the mean synechial/ apposition angle closure, there were significant differences between the two groups ( $p<0.0001$, respectively). Kaplan-Meier analysis revealed that the cumulative probability of success in phaco-GSL group was higher $(p=0.0051)$. They concluded that when compared to trabeculectomy, combined phacoemulsification and viscogoniosynechialysis resulted in a lower requirement for glaucomatous medications, a greater improvement in best corrected visual acuity, and a higher cumulative probability that treatment would be successful among patients with PACG and coexisting cataract. Hence, phaco-GSL can be considered as an alternative when the synechial closure is extensive and IOP is not controlled. 


\section{CLEAR LENS EXTRACTION AS SUBSEQUENT TREATMENT OPTION AFTER PERIPHERAL IRIDOTOMY IN PACG EYES WITHOUT CATARACT}

It is often easier to justify lens extraction in PACG eyes with co-existing cataract, while it is always more controversial when there is no co-existing cataract. Tham et al., in their randomized controlled trial, compared the clinical effects of clear lens extraction versus trabeculectomy with mitomycin $\mathrm{C}$ in treating medically uncontrolled iridotomized PACG eyes [29]. This showed that clear lens extraction alone could reduce the mean IOP by $34 \%$ (reducing mean pre-operative IOP of $24.1 \mathrm{mmHg}$ to $15.9 \mathrm{mmHg}$ post-operatively) and reduce the mean number of drugs by $60.4 \%$ (reducing from 3.69 drugs pre-operatively to 1.46 drugs post-operatively), over 24 months [29]. Whereas, trabeculectomy could reduce the mean tIOP by $36 \%$, (reducing from the mean pre-operative IOP of 24.8 $\mathrm{mmHg}$ to $15.8 \mathrm{mmHg}$ post-operatively) and reduce the mean number of drugs by $88.4 \%$ (reducing from 3.46 drugs preoperatively to 0.40 drugs post-operatively), over 24 months [29]. When compared with lens extraction, trabeculectomy resulted in an additional $0.7 \mathrm{mmHg}$ decrease in mean IOP $(p>/=0.05)$ and a further reduction of 1.1 drugs $(p<0.001)$ over 24 months [29].

From the above study, trabeculectomy with mitomycin-C appeared to be more effective in controlling IOP and reducing dependency on drugs, when compared with clear lens extraction alone. However, within the first 24 months of follow up, $45.8 \%$ of eyes in the trabeculectomy group experienced surgical complications including the development of visually significant cataract (33.3\%), on the other hand only $3.8 \%$ of eyes in clear lens extraction group has a surgical complication. Besides the 8 eyes (33.3\%) that developed cataract in the trabeculectomy group, two eyes (8.3\%) had overdrainage managed with conservative methods, one eye (4.2\%) had hypotony that required reforming of the anterior chamber and subsequent revision of trabeculectomy, and two eyes $(8.3 \%)$ had conjunctival wound leak that were managed with aqueous suppression and eye patching. In the lens extraction group, only one eye (3.8\%) had zonular dehiscence during surgery that required capsular tension ring implantation.

During 2 years after surgery, only $19.2 \%$ eyes in the clear lens extraction group, required subsequent trabeculectomy, while in trabeculectomy group, $25.0 \%$ needed additional surgery (including needling revision of the bleb and cataract extraction). The authors concluded that trabeculectomy with Mytomycin-C resulted in significantly more surgical complications than clear lens extraction in treating medically uncontrolled iridotomized PACG eyes without cataract.

These suggested that clear lens extraction might be an alternative to filtration surgery as an initial surgical option in treating medically uncontrolled iridotomized PACG eyes without co-existing cataract. Clear lens extraction may be preferred in patients who do not accept the risk of filtration surgery or are very vulnerable to, the complications of filtration surgery, on the other hand, if drug reduction is of high priority, trabeculectomy should be more favourable.

\section{ROLE OF LENS EXTRACTION IN ACUTE PRIMARY ANGLE CLOSURE}

Lens extraction at the acute phase of acute primary angle closure (APAC) can be very technically demanding and challenging, and it can also be associated with significant complication risk.

After IOP control and symptomatic relief by medications or ALPI, the following treatment in APAC aims to prevent progression to chronic form of PACG, and to prevent recurrence. Although LPI is often effective, a study conducted by Lam et al. suggested that lens extraction could be more effective in preventing the progression to chronic PACG [30]. Among the APAC eyes which received early lens extraction, only $3.2 \%$ had IOP $>21 \mathrm{mmHg}$ in follow up at 18 months, which appeared to be more favourable when compared with $46.7 \%$ in eyes after LPI [30].

The number of glaucoma medications was significantly fewer after lens extraction than LPI ( 0.03 vs 0.90 respectively, $p<0.0001$ ) at 18 months [30]. The authors concluded that early lens extraction, after initial control of IOP and symptoms, could be a very effective treatment in APAC, so as to prevent its progression to the chronic form.

The role of ALPI as initial management of APAC should also be considered. In the study by Rosman et al., [31] they compared the long-term clinical course of PACG patients in New York and Singapore. In the New York cohort, ALPI was performed to eliminate appositional angle closure after LPI; while in the Singapore cohort, patients received filtration surgery instead of ALPI when IOP became uncontrolled after LPI. Under these different management approaches, $31.3 \%$ of eyes in the New York cohort underwent filtration surgery during follow up, compared with 53.0\% in the Singapore cohort [31].

However, the best timing for 'early' lens extraction after APAC still remains uncertain. On one hand, theoretically 
speaking, the earlier the lens extraction being done, the lower the chance of further PAS formation, and progression to chronic PACG. On the other hand, earlier lens extraction can be more technically challenging and bears a greater risk of complications. Some surgeons prefer to perform 'early' lens extraction at about 1 month after APAC, but this practice has yet to be properly evaluated.

\section{CONCLUSION}

Trabeculectomy is effective in lowering IOP in PACG. However, trabeculectomy is associated with a higher risk of post-operative cataract development or progression, which will in turn lead to a shallower anterior chamber and a more narrow drainage angle. On the other hand, cataract extraction or clear lens extraction can significantly deepen the anterior chamber, open up the drainage angle, and thereby reverse the anatomical predisposition to angle closure. IOP control can also be improved after lens extraction in PACG eyes, and therefore it can be considered as a potential surgical option for PACG. Lens extraction is associated with significantly lower risk of surgical complications and thus a fewer subsequent surgical interventions to handle such complications. Cataract extraction or even clear lens extraction, may be considered in the management of PACG, especially if the patient cannot accept the risk of complications of filtration surgery, or if the diseased eye is vulnerable to the greater risk of combined surgery. Early lens extraction can also be considered in APAC to prevent the progression to chronic PACG significantly, however the best timing for surgery is yet to be determined.

\section{FINANCIAL DISCLOSURES /ACKNOWLEDGEMENTS}

The authors have no relevant affiliations or financial involvement with any organization or entity with a financial interest in or financial conflict with the subject matter or materials discussed in the manuscript. This includes employment, consultancies, honoraria, stock ownership or options, expert testimony, grants or patents received or pending, or royalties.

No writing assistance was utilized in the production of this manuscript.

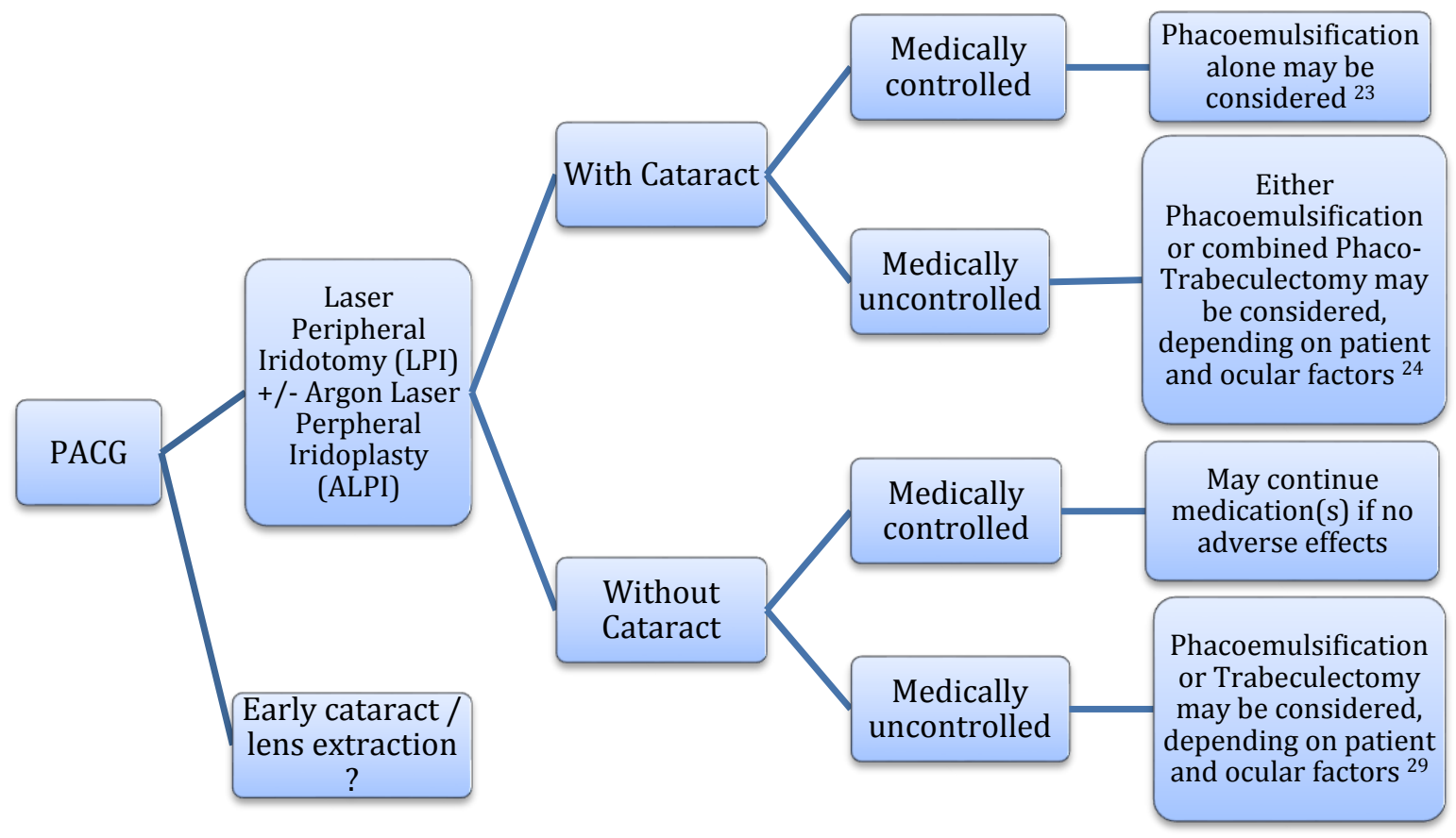

Fig. (1). summarizes the suggested roles of phacoemulsification, combined phacotrabeculectomy, and trabeculectomy alone, in the surgical management algorithm in PACG.

\section{DISCLOSURE}

Part of this article has been previously published in Expert Review of Ophthalmology, Volume 9, Issue 1, 2014, pages 1-5 DOI:10.1586/17469899.2014.864235. 


\section{CONFLICT OF INTEREST}

The authors confirm that this article content has no conflict of interest.

\section{ACKNOWLEDGEMENTS}

Declared none.

\section{REFERENCES}

[1] Schwartz LW, Rodrigues MM, Spaeth GL, Streeten B, Douglas C. Argon laser iridotomy in the treatment of patients with primary angleclosure or pupillary block glaucoma: a clinicopathologic study. Ophthalmology 1978; 85(3): 294-309. [http://dx.doi.org/10.1016/S0161-6420(78)35671-2] [PMID: 566411]

[2] Ritch R, Tham CC, Lam DS. Long-term success of argon laser peripheral iridoplasty in the management of plateau iris syndrome. Ophthalmology 2004; 111(1): 104-8. [http://dx.doi.org/10.1016/j.ophtha.2003.05.001] [PMID: 14711720]

[3] Ritch R, Tham CC, Lam DS. Argon laser peripheral iridoplasty (ALPI): an update. Surv Ophthalmol 2007; 52(3): $279-88$. [http://dx.doi.org/10.1016/j.survophthal.2007.02.006] [PMID: 17472803]

[4] Salmon JF. The role of trabeculectomy in the treatment of advanced chronic angle-closure glaucoma. J Glaucoma 1993; 2(4): 285-90. [http://dx.doi.org/10.1097/00061198-199300240-00011] [PMID: 19920534]

[5] Sihota R, Gupta V, Agarwal HC. Long-term evaluation of trabeculectomy in primary open angle glaucoma and chronic primary angle closure glaucoma in an Asian population. Clin Exp Ophthalmol 2004; 32(1): 23-8. [http://dx.doi.org/10.1046/j.1442-9071.2004.00752.x] [PMID: 14746586]

[6] Tsai HY, Liu CJ, Cheng CY. Combined trabeculectomy and cataract extraction versus trabeculectomy alone in primary angle-closure glaucoma. Br J Ophthalmol 2009; 93(7): 943-8. [http://dx.doi.org/10.1136/bjo.2008.151803] [PMID: 19383599]

[7] Chen YH, Lu DW, Cheng JH, Chen JT, Chen CL. Trabeculectomy in patients with primary angle-closure glaucoma. J Glaucoma 2009; 18(9): 679-83. [http://dx.doi.org/10.1097/IJG.0b013e31819c4a07] [PMID: 20010247]

[8] Lee DA, Brubaker RF, Ilstrup DM. Anterior chamber dimensions in patients with narrow angles and angle-closure glaucoma. Arch Ophthalmol 1984; 102(1): 46-50. [http://dx.doi.org/10.1001/archopht.1984.01040030030029] [PMID: 6703966]

[9] Lowe RF. Anterior lens curvature. Comparisons between normal eyes and those with primary angle-closure glaucoma. Br J Ophthalmol 1972; 56(5): 409-13.

[http://dx.doi.org/10.1136/bjo.56.5.409] [PMID: 5044101]

[10] Lowe RF. Acute angle closure glaucoma and the crystalline lens. Aust J Ophthalmol 1973; 1(3): 89-94. [http://dx.doi.org/10.1111/j.1442-9071.1973.tb00111.x] [PMID: 4804953]

[11] Lowe RF, Clark BA. Radius of curvature of the anterior lens surface. Correlations in normal eyes and in eyes involved with primary angleclosure glaucoma. Br J Ophthalmol 1973; 57(7): 471-4. [http://dx.doi.org/10.1136/bjo.57.7.471] [PMID: 4725855]

[12] Lowe RF, Clark BA. Posterior corneal curvature. Correlations in normal eyes and in eyes involved with primary angle-closure glaucoma. Br J Ophthalmol 1973; 57(7): 464-70. [http://dx.doi.org/10.1136/bjo.57.7.464] [PMID: 4725854]

[13] Nagataki S, Lee DA, Iri M. A simple geometrical method of measuring anterior chamber dimensions. Invest Ophthalmol Vis Sci 1984; 25(10): 1192-7.

[PMID: 6480295]

[14] Tomlinson A, Leighton DA. Ocular dimensions in the heredity of angle-closure glaucoma. Br J Ophthalmol 1973; 57(7): 475-86. [http://dx.doi.org/10.1136/bjo.57.7.475] [PMID: 4725856]

[15] Tham CC, Leung DY, Kwong YY, Li FC, Lai JS, Lam DS. Effects of phacoemulsification versus combined phacotrabeculectomy on drainage angle status in primary angle closure glaucoma (PACG). J Glaucoma 2010; 19(2): 119-23. [PMID: 19373107]

[16] Park SW, Heo H, Yang KJ. Comparison of ultrasound biomicroscopic changes after glaucoma triple procedure and trabeculectomy in eyes with primary angle closure glaucoma. J Glaucoma 2009; 18(4): 311-5. [http://dx.doi.org/10.1097/IJG.0b013e318184568e] [PMID: 19365197]

[17] Baig N, Kam K-W, Tham CC, Ritch R. Lens extraction: where does it stand in the primary angle closure glaucoma management algorithm? Expert Rev Ophthalmol 2014; 9(1): 1-5. [http://dx.doi.org/10.1586/17469899.2014.864235]

[18] Tham CC, Baig N, Leung DY. The role of ens extraction in primary angle-closure glaucoma. In: Shaaraway TM, Hitchings RA, Sherwood 
MB, Crowston JG, Eds. Glaucoma. $2^{\text {nd }}$ ed. Philadelphia: Saunders Ltd. 2015; pp. 999-1004. [http://dx.doi.org/10.1016/B978-0-7020-5193-7.00103-5]

[19] Wishart PK, Atkinson PL. Extracapsular cataract extraction and posterior chamber lens implantation in patients with primary chronic angleclosure glaucoma: effect on intraocular pressure control. Eye (Lond) 1989; 3(Pt 6): 706-12. [http://dx.doi.org/10.1038/eye.1989.109] [PMID: 2630350]

[20] Lai JS, Tham CC, Chan JC. The clinical outcomes of cataract extraction by phacoemulsification in eyes with primary angle-closure glaucoma (PACG) and co-existing cataract: a prospective case series. J Glaucoma 2006; 15(1): 47-52. [http://dx.doi.org/10.1097/01.ijg.0000196619.34368.0a] [PMID: 16378018]

[21] Azuara-Blanco A, Burr JM, Cochran C, et al. The effectiveness of early lens extraction with intraocular lens implantation for the treatment of primary angle-closure glaucoma (EAGLE): study protocol for a randomized controlled trial. Trials 2011; $12: 133$. [http://dx.doi.org/10.1186/1745-6215-12-133] [PMID: 21605352]

[22] Man X, Chan NC, Baig N, et al. Anatomical effects of clear lens extraction by phacoemulsification versus trabeculectomy on anterior chamber drainage angle in primary angle-closure glaucoma (PACG) patients. Graefes Arch Clin Exp Ophthalmol 2015; 253(5): 773-8. [http://dx.doi.org/10.1007/s00417-015-2936-z] [PMID: 25644619]

[23] Tham CC, Kwong YY, Leung DY, et al. Phacoemulsification versus combined phacotrabeculectomy in medically controlled chronic angle closure glaucoma with cataract. Ophthalmology 2008; 115(12): 2167-2173.e2. [http://dx.doi.org/10.1016/j.ophtha.2008.06.016] [PMID: 18801576]

[24] Tham CC, Kwong YY, Leung DY, et al. Phacoemulsification versus combined phacotrabeculectomy in medically uncontrolled chronic angle closure glaucoma with cataracts. Ophthalmology 2009; 116(4): 725-31 [http://dx.doi.org/10.1016/j.ophtha.2008.12.054]

[25] Tham CC, Kwong YY, Leung DY, et al. Phacoemulsification vs phacotrabeculectomy in chronic angle-closure glaucoma with cataract: complications. Arch Ophthalmol 2010; 128(3): 303-11. [http://dx.doi.org/10.1001/archophthalmol.2010.12] [PMID: 20212200]

[26] Teekhasaenee C, Ritch R. Combined phacoemulsification and goniosynechialysis for uncontrolled chronic angle-closure glaucoma after acute angle-closure glaucoma. Ophthalmology 1999; 106(4): 669-74. [http://dx.doi.org/10.1016/S0161-6420(99)90149-5] [PMID: 10201585]

[27] Canlas OA, Ishikawa H, Liebmann JM, Tello C, Ritch R. Ultrasound biomicroscopy before and after goniosynechialysis. Am J Ophthalmol 2001; 132(4): 570-1. [http://dx.doi.org/10.1016/S0002-9394(01)01055-8] [PMID: 11589882]

[28] Tang Y, Qian S, Wang J, et al. Effects of combined phacoemulsification and viscogoniosynechialysis versus trabeculectomy in patients with primary angle-closure glaucoma and coexisting cataract. Ophthalmologica 2012; 228(3): 167-73. [http://dx.doi.org/10.1159/000338241] [PMID: 22739037]

[29] Tham CC, Kwong YY, Baig N, Leung DY, Li FC, Lam DS. Phacoemulsification versus trabeculectomy in medically uncontrolled chronic angle-closure glaucoma without cataract. Ophthalmology 2013; 120(1): 62-7. [http://dx.doi.org/10.1016/j.ophtha.2012.07.021] [PMID: 22986111]

[30] Lam DS, Leung DY, Tham CC, et al. Randomized trial of early phacoemulsification versus peripheral iridotomy to prevent intraocular pressure rise after acute primary angle closure. Ophthalmology 2008; 115(7): 1134-40. [http://dx.doi.org/10.1016/j.ophtha.2007.10.033] [PMID: 18164064]

[31] Rosman M, Aung T, Ang LP, Chew PT, Liebmann JM, Ritch R. Chronic angle-closure with glaucomatous damage: long-term clinical course in a North American population and comparison with an Asian population. Ophthalmology 2002; 109(12): $2227-31$. [http://dx.doi.org/10.1016/S0161-6420(02)01275-7] [PMID: 12466163]

Received: July 26, 2015

Revised: August 18, 2015

Accepted: August 18, 2015

(C) Baig et al.; Licensee Bentham Open.

This is an open access article licensed under the terms of the Creative Commons Attribution-Non-Commercial 4.0 International Public License (CC BY-NC 4.0) (https://creativecommons.org/licenses/by-nc/4.0/legalcode), which permits unrestricted, non-commercial use, distribution and reproduction in any medium, provided the work is properly cited. 